\title{
Comparison of cardiovascular risk calculators in patients with diabetes
}

\author{
Luana Karem Ribeiro ${ }^{1,2 *}$ (1), Welma Wildes Amorim ${ }^{3}$ (1), Igor Thiago Alvin Cardoso ${ }^{3}$ (D), \\ Wester Silva Vieira ${ }^{3}$, Clavdia Nicolaevna Kochergin ${ }^{1}$ (D), Danielle Souto de Medeiros ${ }^{1,2}$ (D), \\ Daniela Arruda Soares ${ }^{1,2}$ (D), José Andrade Louzado ${ }^{1}$ (D), Kelle Oliveira Silva ${ }^{1}$ (1),

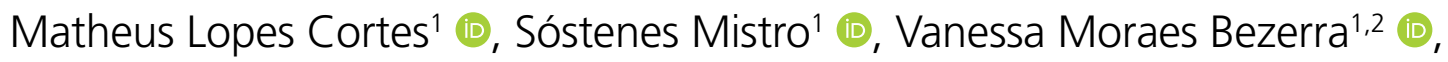 \\ Marcio Galvão Oliveira' ${ }^{1,2}$
}

\begin{abstract}
SUMMARY
OBJECTIVE: Cardiovascular risk stratification is an important clinical practice to estimate the severity of cardiovascular disease in patients with type 2 diabetes. This study aimed to compare the stratification of global cardiovascular risk with the specific risk stratification for patients with type 2 diabetes, seen at specialized outpatient clinics, and to evaluate possible differences in diagnoses and treatments. METHODS: A total of 122 patients with type 2 diabetes treated at two specialized outpatient clinics, from 2017 to 2019 , were studied. The cardiovascular risk stratification calculators, global risk score, Cardiovascular Risk Stratification Calculator, and United Kingdom Prospective Diabetes Study-Risk Engine, were used to calculate the risk of death from cardiovascular disease. The agreement between these calculators was analyzed using the kappa index. The indications for the use of statins and acetylsalicylic acid for the group studied were evaluated according to the Brazilian Diabetes Society Guideline.

RESULTS: There was a low degree of agreement among the three risk calculators. The global risk score calculator showed insignificant agreement with the Cardiovascular Risk Stratification Calculator (kappa=0.0816; $p=0.0671$ ). There was no agreement between the global risk score calculator and United Kingdom Prospective Diabetes Study-Risk Engine (kappa=-0.099), or between the Cardiovascular Risk Stratification Calculator and United Kingdom Prospective Diabetes Study-Risk Engine (kappa=-0.0095).

CONCLUSION: The substantial disagreements among the cardiovascular risk calculators may lead to different diagnoses and may consequently influence therapeutic strategies. The findings herein highlight the need for specific validated cardiovascular risk calculators for patients with DM2 that can reliably estimate risk in these individuals.

KEYWORDS: Diabetes mellitus. Cardiovascular diseases. Cardiovascular risk.
\end{abstract}

\section{INTRODUCTION}

Cardiovascular risk stratification is an important clinical practice to determine the severity of cardiovascular disease, especially in asymptomatic patients who are more susceptible to clinical complications, such as acute coronary syndromes, strokes, transient ischemic attacks, and peripheral arterial disease $^{1}$. To help health professionals analyze their patients' risks quickly and easily to propose therapeutic measures, several cardiovascular risk calculators have been developed, all based on different risk factors. Some of these tools are used for the

\footnotetext{
'Universidade Federal da Bahia, Multidisciplinary Health Institute - Vitória da Conquista (BA), Brazil.

${ }^{2}$ Universidade Federal da Bahia, Multidisciplinary Health Institute, Postgraduate Program in Collective Health - Vitória da Conquista (BA), Brazil.

${ }^{3}$ Universidade Estadual do Sudoeste da Bahia, Medical School - Vitória da Conquista (BA), Brazil.

*Corresponding author: lua.karem.ribeiro@gmail.com

Conflicts of interest: the authors declare there are no conflicts of interest. Funding: This study was funded by the Medtronic Foundation.

Received on August 17, 2020. Accepted on October 31, 2020.
} 
general population, whereas others are used for specific populations, such as those with diabetes mellitus.

Type 2 diabetes mellitus (DM2) is one of the main risk factors for cardiovascular diseases ${ }^{2}$, increasing cardiovascular morbidity and mortality 2-4-fold in relation to individuals without DM2 ${ }^{3}$. The diagnosis of DM2 was considered equivalent to a high cardiovascular risk ${ }^{4}$ and, therefore, cardiovascular risk stratification guidelines and instruments traditionally used in clinical practice ${ }^{5}$ automatically consider patients with DM2 to be at high cardiovascular risk. Nevertheless, not all patients with DM2 have the same degree of cardiovascular risk ${ }^{6}$.

Stratification of cardiovascular risk appropriate to patients with diabetes can improve the accuracy of prediction of subclinical cardiovascular disease, silent ischemia, and future cardiovascular events. It can also prevent unnecessary use of aggressive treatment in low-risk patients that might otherwise increase the risk of adverse events and high treatment costs. For these reasons, cardiovascular prevention strategies must be individualized according to cardiovascular risk, whereas intensified treatment must be reserved for individuals at higher risk ${ }^{7}$.

This article aims to compare global cardiovascular risk stratification with the specific cardiovascular risk stratification for patients with DM2, who attended two specialized outpatient clinics, and to evaluate possible differences in diagnoses and treatments. It also allows for the comparison of national calculators - global risk score (GRS) and cardiovascular risk stratification calculator (ER Calculator) - with the United Kingdom Prospective Diabetes Study-Risk Engine (UKPDS-RE) calculator and for an evaluation of the effectiveness of these calculators.

\section{METHODS}

\section{Study design and population}

This is a cross-sectional study derived from a cohort entitled HealthRise Vitória da Conquista, an intervention project designed to improve the control of diabetes and hypertension in primary care and specialized outpatient clinics in Vitória da Conquista City, Bahia State. The study included all patients with DM2 at two specialized outpatient clinics, referred by the city's primary health care facilities, from 2017 to 2019. Patients who had cardiovascular disease at the beginning of the study (high cardiovascular risk) were excluded.

\section{Data collection}

Patients examined between July 2018 and July 2019 were considered. The electronic medical records of patients examined at medical specialty clinics were reviewed. Patient data (variables of interest) selected from the electronic forms were recorded in an online questionnaire specially designed for this study.

\section{Measures and definitions}

The following variables were analyzed: a) Demographic: sex, age, marital status, religion, skin color, education, profession, and current economic situation; b) Anthropometric: weight, height, body mass index (BMI), waist circumference, hip circumference, and waist/hip ratio (WHR); c) Clinical: systolic blood pressure (SBP), diastolic blood pressure (DBP), ankle-brachial index (ABI), classic symptoms of diabetes (polyuria/ urinary incontinence, urgency, polydipsia, polyphagia, and weight loss); d) Clinical features (hypoglycemia, ketoacidosis, hyperglycemia, and infection), time of diagnosis of DM and types of treatment, initial clinical presentation (diabetic ketoacidosis, hyperosmolar hyperglycemic state, asymptomatic laboratory findings); and e) Personal details and complications (retinopathy, nephropathy, peripheral neuropathy, autonomic neuropathy, infarction, stroke, carotid disease, diabetic foot, limb amputation, angina, atrial fibrillation, and metabolic syndrome), medications, and life habits (smoking).

\section{Stratified cardiovascular risk}

Age was stratified into four categories, and the following variables were dichotomized: sex, alcohol consumption, diagnosis, presence of treated systemic arterial hypertension $(\mathrm{SAH})$, classic symptoms of DM, clinical complications, initial clinical presentation, comorbidities, use of acetyl salicylic acid and statins, clinical complications, presence of personal and family comorbidities, and family history of premature coronary artery disease, as defined by the Brazilian Diabetes Society

Regarding smoking habits, patients were categorized as current smoking (defined when the last episode occurred less than a year before the moment of stratification), non-smoker, and former smoker?

The following continuous variables were studied: time of diagnosis of DM and SAH, body mass index (BMI), WHR, SBP, DBP, fasting glucose levels, postprandial glucose levels, HbA1c level, creatinine clearance, total cholesterol (TC), high density lipoproteins (HDL), low density lipoproteins (LDL), and triglycerides. The stratified cardiovascular risk from the calculators was classified as low, intermediate, high, based on the risk score of each calculator used.

\section{Cardiovascular risk scoring models}

Risk stratification calculators were used to estimate the risk of death from coronary heart disease, non-fatal infarction, angina, fatal or non-fatal ischemic or hemorrhagic stroke, transient 
ischemic attack, intermittent claudication, and heart failure over 10 years.

To calculate the cardiovascular risk (CVR) of patients with DM2, risk calculators were selected that derive from the Framingham models ${ }^{1}$ and that use traditional risk factors: the GRS of the Brazilian Society of Cardiology $(\mathrm{SBC})^{8}$ and the UKPDS Risk Engine 2.0 ${ }^{9}$, specific for patients with DM. We also used the CVR stratification calculator (ER Calculator) prepared by the Department of Atherosclerosis of the Brazilian Society of Cardiology, which is based on the Update of the Brazilian Guidelines on Dyslipidemia and Prevention of Atherosclerosis $2017^{10}$. This score also uses non-traditional risk factors (RF).

Indications for the use of statins and acetylsalicylic acid (ASA) for the group studied were also evaluated from the CVR stratifications, according to the Brazilian Diabetes Society (Sociedade Brasileira de Diabetes - SBD) Directive ${ }^{7}$. Statin use and dose was based on LDL and n-HDL targets for each risk group. ASA use is indicated for DM patients considered high risk, without atherosclerotic disease, when they are older than 65 and present a low risk of bleeding; in very high-risk patients with atherosclerotic disease, it is indicated for secondary prevention.

These scoring models were selected because they have already been used in cohort studies with robust samples ${ }^{8,911-14}$, including patients with DM.

\section{Data analysis}

CVR percentages were calculated considering the age groups for each calculator to estimate the development of cardiovascular disease (CVD) over 10 years. Patients with missing data were excluded when calculating the risk for each model. STATA 15.0 software was used for statistical analysis. P-value $<0.05$ was accepted as significant. Descriptive statistics were used for frequencies, means, and standard deviations. Pearson's $\chi^{2}$, Mann-Whitney U, and Fisher's exact tests were used to assess demographic characteristics, risk factors, and 10-year risks. Pearson's $\chi^{2}$ analysis was used to determine the relation between 10 -year risk and age groups. The $\chi^{2}$ test was performed, and the linear association value was considered when the expected values less than five in a column were greater than $25 \%$. The Kruskal-Wallis test was used to assess the relationship between CVD risk, alcohol use, and physical activity habits.

To assess the degree of agreement among the SBC global risk score calculators, the kappa agreement coefficient was calculated.

\section{Ethical considerations}

The requirement for consent was waived because of the retrospective nature of the study. The work was approved by a local research ethics committee.

\section{RESULTS}

During the study period, 1,276 patients were followed up. Of these, 122 were eligible, 64 (52.46\%) of whom were women. The median age was 59 years old, and those over 60 years old comprised $48.36 \%$ of the population. Regarding skin color, 47 (38.52\%) declared themselves as pardo and 16 (13.11\%) self-identified as black. In general, the subjects had low levels of education, with 11 (9.02\%) reporting being illiterate. Regarding the economic situation, 44 (36.07\%) patients were salaried, 11 (9.02\%) were unemployed, and $44(36.07 \%)$ were retired or received some pension.

When analyzing risk factors for CVD, stratified by sex, we found that the average time since diagnosis of DM in the overall population was five years; however, $50.41 \%$ of the population had been diagnosed within five years. Overall, 96 (79\%) were hypertensive, 53 (55.21\%) of whom were women.

Regarding DM treatment, 100 (82.64\%) patients used oral antihyperglycemic agents and only $23(18.85 \%)$ used insulins. Lipid-lower drugs (58.20\%) and antiplatelet drugs (38.52\%) showed a high frequency of use with little difference between genders. Peripheral neuropathy was the most frequent complication of DM in the study group, with 34 (27.87\%) affected patients (Table 1).

When assessing the 10-year risk grouping on the selected calculators, stratified by age, the global risk score categorized high risk CVR in 105 (86.07\%) patients. Of these, 57 $(54.29 \%)$ older than 60 years $(\mathrm{p}<0.05)$. There was a high proportion of CVR of $47.62 \%$ among men and $52.38 \%$ for women; however, there was no significant difference for this calculator $(\mathrm{p}=0.430)$.

The calculator of the Brazilian Diabetes Society classified $111(90.98 \%)$ patients with high cardiovascular risk, 55 $(49.55 \%)$ of whom were over 60 years old. When stratified by sex, a high CVR was also observed between groups, in 55 $(52.38 \%)$ women and $50(47.62 \%)$ men. Stratification for both sex and age did not show a significant difference for this calculator $(\mathrm{p}=0.940)$.

The UKPDS-RE, unlike the other calculators, classified 77 (63.11\%) and 30 (24.59\%) patients, as low and intermediate CVR, respectively $(\mathrm{p}<0.001)$. Of the low CVR group, $34(44.16 \%)$ were younger adults (31-50 years old). The proportions between men and women classified by this calculator were similar (Table 2).

There was a low level of agreement between risk calculators using the kappa index. When comparing GRS calculators with the ER Calculator, there was insignificant agreement (kappa $=0.0816$; $\mathrm{p}=0.067)$. No agreement was observed between the GRS calculator and UKPDS-RE (kappa=-0.099), or between the ER Calculator and UKPDS-RE (kappa=-0.0095). 
Table 1. Risk factors for cardiovascular diseases and metabolic control parameters in people with type II diabetes, Vitória da Conquista City, Bahia State, 2017-2019.

\begin{tabular}{|c|c|c|c|c|}
\hline & Total $(n=122)$ & Women $(n=64)$ & Men $(n=58)$ & n-value \\
\hline & $n$ & $\mathrm{n}(\%)$ & $\mathrm{n}(\%)$ & $p$-value \\
\hline DM duration & & & & \\
\hline$<5$ years & $61(50.41)$ & $29(47.54)$ & $32(52.46)$ & \\
\hline $5-10$ years & $16(13.22)$ & $9(56.25)$ & $7(43.75)$ & \\
\hline $10-20$ years & $27(22.31)$ & $17(62.96)$ & $10(37.04)$ & $0.558^{*}$ \\
\hline$>20$ years & $17(14.05)$ & $8(47.06)$ & $9(52.94)$ & \\
\hline Presence of SAH & & & & \\
\hline No & $26(21.31)$ & $11(42.31)$ & $15(57.69)$ & \\
\hline Yes & 96 (78.69) & $53(55.21)$ & $43(44.79)$ & 0.243 \\
\hline SAH duration & & & & \\
\hline$<5$ years & $26(24.76)$ & $7(26.92)$ & $19(73.08)$ & \\
\hline $5-10$ years & $18(17.14)$ & $11(61.11)$ & $7(38.89)$ & ค 001 * \\
\hline $10-20$ years & $33(31.43)$ & $26(78.79)$ & $7(21.21)$ & 0.001 \\
\hline$>20$ years & $28(26.67)$ & $15(15.7)$ & $13(12.3)$ & \\
\hline DM treatment & & & & \\
\hline ADO (\%) & $100(82.64)$ & $54(54.00)$ & $46(46.00)$ & $0.353^{*}$ \\
\hline Insulin & $23(18.85)$ & $13(56.52)$ & $10(43.48)$ & $0.665^{*}$ \\
\hline ADO + Insulin & $15(12.40)$ & $9(60.0)$ & $6(40.0)$ & $0.511^{*}$ \\
\hline Non-diabetic drugs & & & & \\
\hline Antihypertensives & $106(86.89)$ & $59(55.66)$ & $47(44.34)$ & $0.068^{*}$ \\
\hline Lipid-lowering drugs & $71(58.20)$ & $40(56.34)$ & $31(43.66)$ & $0.311^{*}$ \\
\hline Antiplatelet & $47(38.52)$ & $32(68.09)$ & $15(31.91)$ & $0.006^{*}$ \\
\hline Anti-arrhythmic & $3(2.46)$ & $2(66.7)$ & $1(33.33)$ & $1.00^{* *}$ \\
\hline DM complications & & & & \\
\hline Retinopathy & $34(27.87)$ & $17(50)$ & $17(50)$ & $0.735^{*}$ \\
\hline Nephropathy & $12(9.84)$ & $4(33.33)$ & $8(33.33)$ & $0.162^{*}$ \\
\hline Peripheral neuropathy & $25(20.66)$ & $14(56)$ & $11(44)$ & $0.658^{*}$ \\
\hline Autonomic neuropathy & $4(3.28)$ & $2(50)$ & $2(50)$ & $1.00^{* *}$ \\
\hline Diabetic foot & $8(6.56)$ & $3(37.5)$ & $5(62.5)$ & $0.476^{\star}$ \\
\hline Amputation of limbs & $4(3.28)$ & $1(25)$ & $3(75)$ & $0.345^{*}$ \\
\hline Angina & $10(8.20)$ & $8(80)$ & $2(20)$ & $0.099^{* *}$ \\
\hline $\mathrm{AF}$ & $2(1.64)$ & $0(0)$ & $2(100)$ & $0.224^{* *}$ \\
\hline Smokinga & & & & \\
\hline Non-smoker & 89 (72.95) & $47(52.81)$ & $42(47.19)$ & \\
\hline Former smoker & $16(13.11)$ & $9(56.25)$ & $7(43.75)$ & $0.833^{*}$ \\
\hline Smoker & $16(13.11)$ & $7(43.75)$ & $9(56.25)$ & \\
\hline Alcohol use ${ }^{a}$ & & & & \\
\hline No ethanol & $86(70.49)$ & $52(60.47)$ & 34 (39.53) & \\
\hline Alcoholic & $24(19.67)$ & $5(20.83)$ & $19(79.17)$ & $0.003^{*}$ \\
\hline Former alcoholic & $11(9.02)$ & $6(54.55)$ & $5(45.45)$ & \\
\hline Physical Activity Practicea & & & & \\
\hline No & 89 (72.95) & $48(55.93)$ & $41(46.07)$ & 0 \\
\hline Yes & $32(26.23)$ & $15(46.88)$ & $17(53.12)$ & 0.493 \\
\hline $\mathrm{BMI}^{\mathrm{a}}$ & & & & \\
\hline Underweight & $5(4.55)$ & $3(60)$ & $2(40)$ & \\
\hline Normal & $22(20)$ & $5(22.73)$ & $17(77.27)$ & \\
\hline Overweight & $31(28.18)$ & $14(45.16)$ & $17(54.84)$ & 0.05 \\
\hline Obesity & $52(47.27)$ & $34(65.38)$ & $18(34.62)$ & \\
\hline SBP (average) & $140( \pm 21.19)$ & $150( \pm 27.82)$ & $140( \pm 26.2)$ & $0.2015^{* \star *}$ \\
\hline DBP (average) & $86( \pm 14.13)$ & $85( \pm 14.46)$ & $87( \pm 13.83)$ & $0.8810 * * *$ \\
\hline GJ (average) & $121.5( \pm 69.51)$ & $124.5( \pm 67.37)$ & $116( \pm 72.35)$ & $0.374 * * *$ \\
\hline HBA1C (average) & $7.5( \pm 1.98)$ & $7.55( \pm 1.84)$ & $7.1( \pm 2.14)$ & $0.3363^{* \star *}$ \\
\hline CT (average) & $187.02( \pm 43.33)$ & $183.5( \pm 44.46)$ & $183.5( \pm 41.62)$ & $0.402 * * *$ \\
\hline HDL (average) & $43.5( \pm 11.09)$ & $45( \pm 9.47)$ & $39( \pm 12.02)$ & $0.0009 * \star \star$ \\
\hline LDL1 (average) & $102( \pm 37.93)$ & $101.5( \pm 38.21)$ & $102.5( \pm 37.87)$ & $0.8395^{* * *}$ \\
\hline TG (mean) & $154( \pm 186.37)$ & $140( \pm 117.05)$ & $171.5( \pm 118.10)$ & $0.4527 * * *$ \\
\hline
\end{tabular}


A second analysis was performed based on a grouping of the ER Calculator (ER1). The high-risk and very high-risk categories were grouped into only high risk. In this manner, better agreement was obtained between GRS and ER1 (kappa=0.1545), although it was still statistically insignificant.

Regarding the indication for medication use in the studied population, a large difference was noticed in the indications for statins and ASA. Whereas the GRS calculator and the ER Calculator indicate the use of statins in 105 and 118 patients, respectively, the UKPDS-RE calculator indicates their use only in 15 patients. For ASA, the number of patients referred by the GRS and ER calculators would be more than four times higher than by the UKPDS-RE calculator (Table 3).

\section{DISCUSSION}

Low levels of agreement were observed among the CVR calculators selected in this study. This observation is consistent with results of studies that used other types of calculators ${ }^{15-17}$.

The GRS, recommended by the SBC and derived from the Framingham Heart Study equations ${ }^{18}$, estimates the risk of CVD in 10 years. Using this tool, patients are categorized as low risk $(<5 \%)$, intermediate risk (men with calculated risk $\geq 5$ and $\leq 20 \%$, and women with calculated risk $\geq 5$ and $\leq 10 \%$ ) and high risk (risk calculated $>20 \%$ for men and $>10 \%$ for women over 10 years). Patients classified in the low risk category and who have a family history of premature cardiovascular disease are reclassified as intermediate risk ${ }^{18}$.
The recommendation is that the GRS be used in the initial assessment of individuals who were not included in high-risk conditions ${ }^{18}$. However, the study population in this study is composed exclusively of patients with DM2, which leads most patients to be classified as high CVR. This can lead to more aggressive therapeutic approaches and, consequently, to polypharmacy prescription 5 .

The UKPDS risk engine is a specific risk calculator for type 2 diabetes, based on data from 53,000 patients in the UK Prospective Diabetes Study ${ }^{10}$. This tool provides risk estimates and $95 \%$ confidence intervals for individuals with

Table 3. Indication for the use of statins and acetylsalicylic acid, based on cardiovascular risk by cardiovascular risk calculator: GRS, UKPDS-RE, and ER Calculator, Vitória da Conquista City, Bahia State, 2017-2019.

\begin{tabular}{|c|c|c|}
\hline & No n (\%) & Yes n (\%) \\
\hline \multicolumn{3}{|l|}{ Statin Use } \\
\hline GRS & $17(13.94)$ & 105 (86.06) \\
\hline UKPDS-RE & 107 (87.7) & $15(12.3)$ \\
\hline ER Calculator & $4(3.28)$ & $118(96.72)$ \\
\hline \multicolumn{3}{|l|}{ ASA Use } \\
\hline GRS & 78 (63.93) & $44(36.07)$ \\
\hline UKPDS-RE & $112(91.80)$ & $10(8.20)$ \\
\hline ER Calculator & $76(62.30)$ & $46(37.70)$ \\
\hline
\end{tabular}

GRS: global risk score; ER Calculator: cardiovascular risk stratification calculator; UKPDS-RE: United Kingdom Prospective Diabetes Study-Risk Engine; ASA: acetylsalicylic acid.

Table 2. Grouping of 10-year risks according to models stratified by sex, Vitória da Conquista City, Bahia State, 2017-2019.

\begin{tabular}{|c|c|c|c|c|}
\hline & Total n (\%) & Women n (\%) & Men n (\%) & $\mathrm{p}$-value \\
\hline \multicolumn{5}{|l|}{ GRS } \\
\hline Low & 5 & $4(80)$ & $1(20)$ & \multirow{3}{*}{$0.430^{* *}$} \\
\hline Intermediate & 12 & $5(41.67)$ & $7(58.33)$ & \\
\hline High & 105 & 55 (52.38) & $50(47.62)$ & \\
\hline \multicolumn{5}{|l|}{ ER Calculator } \\
\hline Low & 1 & $1(100)$ & 0 & \multirow{4}{*}{$0.940^{*}$} \\
\hline Intermediate & 3 & $2(66.67)$ & $1(33.33)$ & \\
\hline Very High & 111 & $58(52.25)$ & $53(47.75)$ & \\
\hline Extremely High & 7 & $3(42.86)$ & $4(57.14)$ & \\
\hline \multicolumn{5}{|l|}{ UKPDS - RE } \\
\hline Low & 77 & $41(53.25)$ & 36 (46.75) & \multirow{3}{*}{$0.234^{*}$} \\
\hline Intermediate & 30 & $18(60)$ & $12(40)$ & \\
\hline High & 15 & $5(33.33)$ & $10(66.67)$ & \\
\hline
\end{tabular}

GRS: global risk score; ER Calculator: cardiovascular risk stratification calculator; UKPDS-RE: United Kingdom Prospective Diabetes Study-Risk Engine. *Chi-square test; **Fisher's exact test 
type $2 \mathrm{DM}$ who do not have heart disease. The CVR can be calculated for all patients with DM2, regardless of the time of diagnosis. It uses the following risk factors: age; sex; ethnicity; smoking; presence or absence of atrial fibrillation; and levels of HbA1c, SBP, cholesterol total, and HDL cholesterol. Among the percentages referring to total risk, scores $<10 \%$ indicate low risk, $10-19 \%$ indicates medium risk, and $\geq 20 \%$ indicates high risk ${ }^{10}$.

The agreement between the global risk score and the UKPDS-RE calculators in this study was very low. This can be explained by the fact that these calculators use different risk factors and generate divergent classifications for the same patients. The overestimation of CVD risk that derive from the Framingham calculator compared to the UKPDS-RE demonstrates the importance of using glycated hemoglobin levels to estimate CVD risk in $\mathrm{DM} 2^{8}$.

The ER calculator ${ }^{7}$ is valid for patients with DM onset after 18 years of age; therefore, it is well-suited for this study. Using this calculator, patients with DM were divided into four major categories of cardiovascular risk: low, intermediate, high, and very high, according to age, the presence of risk stratifiers (RS), subclinical atherosclerotic disease, or clinical atherosclerotic disease. The 10-year cardiovascular event rates for low, intermediate, high and very high risk were $<10,10-20,20-30$ and $>0 \%$, respectively.

According to $\mathrm{SBD}^{7}$, the ER calculator is derived from the UKPDS-RE risk score; however, the agreement according to the kappa index was insufficient, suggesting that the ER calculator is not ideal for risk stratification in patients with diabetes. The divergence in the stratification of CVR by these two calculators generated different indications for the use of ASA and statins for the studied group. Therefore, depending on the chosen risk calculator, different diagnostic and therapeutic approaches would be adopted.

The present study allowed us to understand the applicability of national non-specific CVR calculators for diabetic patients with a calculator already validated internationally for this population. It is suggested that, based on this study, other studies may address a larger number of patients to evaluate these calculators, as well as using other types of calculators produced nationwide.

\section{Study limitations}

The present study had a small study population derived from medical specialty clinics; therefore, they represent potentially more severely-affected patients. Nevertheless, the population was quite heterogeneous both in terms of demographics and clinical profiles. It is also important to note that the loss of information when reviewing medical records was substantial.
Another limitation was the use of statins prior to the study period, which might have underestimated cardiovascular risk, specifically in the UKPDS-RE calculator.

\section{CONCLUSIONS}

Important disagreements were observed between the CVR calculators; this can lead to different diagnoses and, consequently, can influence therapeutic strategies. The use of the UKPDS equation made it possible to identify those at high risk for CVD early. This may avoid polypharmacy prescription in patients considered to be at low risk. In this sense, according to our findings, this scale should be considered superior to the other calculators.

Incorporating DM2 as a categorical variable implies that diabetes increases the risk in a similar way, regardless of glycemic control or the duration of diabetes. This work, therefore, emphasizes the need to use specific and validated risk calculators for individuals with a diagnosis of DM2 that can reliably estimate the risk of CVD.

\section{ACKNOWLEDGMENTS}

We are grateful for the support provided by SESI and CEUAS, and to the Master's students of the Collective Health Program/ UFBA. This study was conducted as part of the HealthRise project, led by Abt Associates and the Institute for Health Metrics and Evaluation.

\section{AUTHORS' CONTRIBUTIONS}

LKR: Conceptualization, Data Curation, Formal Analysis, Writing - Original Draft. WWA: Conceptualization, Data Curation, Formal Analysis, Writing - Original Draft. VMB: Conceptualization, Data Curation, Formal Analysis, Writing - Original Draft. MGO: Conceptualization, Data Curation, Formal Analysis, Writing - Original Draft. WSV: Conceptualization, Data Curation. ITAC: Conceptualization, Writing - Original Draft, Writing - Review \& Editing. CNK: Conceptualization, Writing - Original Draft, Writing - Review \& Editing. DSM: Conceptualization, Writing - Original Draft, Writing - Review \& Editing. DAS: Conceptualization, Writing - Original Draft, Writing - Review \& Editing. JAL: Conceptualization, Writing - Original Draft, Writing - Review \& Editing. KOS: Conceptualization, Writing - Original Draft, Writing - Review \& Editing. MLC: Conceptualization, Writing - Original Draft, Writing - Review \& Editing. SM: Conceptualization, Writing - Original Draft, Writing Review \& Editing. 


\section{REFERENCES}

1. D'Agostino RB Sr, Grundy S, Sullivan LM, Wilson P: CHD Risk Prediction Group. Validation of the Framingham coronary heart disease prediction scores: results of a multiple ethnic groups investigation. JAMA. 2001;286(2):180-7. https://doi. org/10.1001/jama.286.2.180

2. Martín-Timón I, Sevillano-Collantes C, Segura-Galindo A, Del Cañizo-Gómez FJ. Type 2 diabetes and cardiovascular disease: have all risk factors the same strength? World J Diabetes. 2014;5(4):444-70. https://doi.org/10.4239/wjd.v5.i4.444

3. Bertoluci MC, Rocha VZ. Cardiovascular risk assessment in patients with diabetes. Diabetol Metab Syndr. 2017;9:25. https://doi.org/10.1186/s13098-017-0225-1

4. Haffner SM, Lehto S, Rönnemaa T, Pyörälä K, Laakso M. Mortality from coronary heart disease in subjects with type 2 diabetes and in nondiabetic subjects with and without prior myocardial infarction. N Engl J Med. 1998;339(4):229-34. https://doi.org/10.1056/NEJM199807233390404

5. Expert Panel on Detection, Evaluation, and Treatment of High Blood Cholesterol in Adults. Executive summary of the third report of The National Cholesterol Education Program (NCEP) expert panel on detection, evaluation, and treatment of high blood cholesterol in adults (Adult Treatment Panel III). 2001;285(19):2486-97. https://doi.org/10.1001/jama.285.19.2486

6. Bulugahapitiya U, Siyambalapitiya S, Sithole J, Idris I. Is diabetes a coronary risk equivalent? Systematic review and meta-analysis. Diabet Med. 2009;26(2):142-8. https://doi. org/10.1111/j.1464-5491.2008.02640.x

7. Bertoluci MC, Moreira RO, Faludi A, Izar MC, Schaan BD, Valerio $\mathrm{CM}$, et al. Brazilian guidelines on prevention of cardiovascular disease in patients with diabetes: a position statement from the Brazilian Diabetes Society (SBD), the Brazilian Cardiology Society (SBC) and the Brazilian Endocrinology and Metabolism Society (SBEM). Diabetol Metab Syndr. 2017;9:53. https://doi. org/10.1186/s13098-017-0251-z

8. Sociedade Brasileira de Cardiologia (SBC). O Cardiômetro. Rio de janeiro: Sociedade Brasileira de Cardiologia; 2015. [cited on Jan. 15, 2020]. Avaliable from: http://www.cardiometro.com.br/

9. Bannister $C A$, Poole $C D$, Jenkins-Jones $S$, Morgan $C L$, Elwyn $\mathrm{G}$, Spasic I, et al. External validation of the UKPDS risk engine in incident type 2 diabetes: a need for new type 2 diabetesspecific risk equations. Diabetes Care. 2014;37(2):537-45. https://doi.org/10.2337/dc13-1159
10. Coleman, R. Stevens, R. Holman, R. Updated UKPDS risk engine that estimates primary and secondary cardiovascular disease risk in people with recently-diagnosed or established type 2 diabetes. Diabetes. 2012;61(Suppl 1):A264. [cited on Sept. 10, 2019]. Available from: http://www.dtu.ox.ac.uk/ Abstracts/DTU_MtgAbs106_Abstract.pdf

11. Alrawahi, AH. Lee, P. Al-Anqoudi, ZAM. Alrabaani, M. Al-Busaidi, A. Almahrouqi, F. et al. Cardiovascular risk prediction model for Omanis with type 2 diabetes. Diabetes Metab Syndr. 2018;12(2):105-10. https://doi.org/10.1016/j. dsx.2017.09.012

12. Alrawahi $A H$, Lee $P$. Validation of the cardiovascular risk model developed for Omanis with type 2 diabetes. Diabetes Metab Syndr. 2018;12(3):387-91. https://doi.org/10.1016/j. dsx.2018.01.004

13. Grossmann V, Schmitt VH, Junger $C$, Munzel T, Wild, PS. Cardiovascular risk stratification in patients with type 2 diabetes. Diabetologe. 2014;10:572-80. https://doi.org/10.1007/ s11428-014-1213-9

14. Chamnan P, Simmons RK, Sharp SJ, Griffin SJ, Wareham NJ. Cardiovascular risk assessment scores for people with diabetes: a systematic review. Diabetologia. 2009;52(10):2001-14. https://doi.org/10.1007/s00125-009-1454-0

15. Coleman RL, Stevens RJ, Retnakaran R, Holman RR. Framingham, SCORE, and DECODE risk equations do not provide reliable cardiovascular risk estimates in type 2 diabetes. Diabetes Care. 2007;30(5):1292-3. https://doi.org/10.2337/dc06-1358

16. Bayındır Çevik A, Özcan Ş, Satman İ. Sensitivity of FRAMINGHAM, PROCAM and SCORE models in Turkish people with Type 2 diabetes: comparison of three cardiovascular risk calculations. Contemp Nurse. 2015;50(2-3):183-95. https://doi.org/10.10 80/10376178.2015.1111153

17. Yew SQ, Chia YC, Theodorakis M. Assessing 10-Year cardiovascular disease risk in malaysians with type 2 diabetes mellitus: Framingham cardiovascular versus United Kingdom prospective diabetes study equations. Asia Pac J Public Health. 2019;31(7):622-32. https://doi. org/10.1177/1010539519873487

18. Simão $A F$, Precoma $D B$, Andrade JP, Correa Filho $H$, Saraiva JFK, Oliveira GMM, et al. I Diretriz brasileira de prevenção cardiovascular. Arq Bras Cardiol. 2013;101(6 Suppl 2):1-63. https://doi.org/10.5935/abc.20135012 